\title{
Ciclismo urbano en Buenos Aires. Movilidades y etnografía
}

\author{
Oscar Ramos Mancilla \\ Instituto de Investigaciones Gino Germani, Facultad de Ciencias Sociales, Universidad de \\ Buenos Aires, Argentina
}

Recibido: 31 de octubre de 2017. Aceptado: 19 de marzo de 2018.

\begin{abstract}
Resumen
En este artículo describo elementos de la práctica del ciclismo urbano en la ciudad de Buenos Aires. Expongo una revisión a los estudios socioculturales en torno al ciclismo urbano y, por otro lado, despliego un ejercicio de etnografía donde expongo algunos aspectos vinculados a la práctica del ciclismo en la ciudad. En ambas partes se retoma el paradigma de las movilidades para guiar las interpretaciones. La información es resultado de un periodo de trabajo de campo centrado en la movilidad activa en la ciudad de Buenos Aires entre el 2016 y 2017. Al realizar la investigación se constató que cualquier práctica de movilidad (en este caso activa) es necesariamente una práctica corporal, la cual genera sentidos y significados, espacios de socialización con quienes se comparte el ciclismo, y se reelaboran los conocimientos de los entornos urbanos por donde se transita. Finalmente, identifiqué la conformación de un espacio de socialización a partir de los paseos intencionales y colectivos que están emergiendo en la ciudad y que suman mayor heterogeneidad al ciclismo urbano.
\end{abstract}

\section{Urban cycling in Buenos Aires. Mobilities and ethnography.}

Palabras clave

Bicicleta Movilidades Etnografía
Buenos Aires

\begin{abstract}
In this paper I approach the practice of urban cycling in the city of Buenos Aires. I review socio-cultural studies on urban cycling and I try to carry out an ethnography exercise to reflect on some aspects which relate to the practice of cycling in the city. In both parts of the paper, the paradigm of mobilities guides the interpretations. The data displayed here is the outcome of a fieldwork period focused on active mobility in the city of Buenos Aires between 2016 and 2017. When carrying out the research, it was noted that every mobility practice (active mobility practice in this case) is necessarily a bodily practice, which generates senses and meanings, as well socialisation spaces which those with whom the cycling is shared, and knowledge about the transited urban
\end{abstract}

Keywords

Bicycle Mobilities Ethnography Buenos Aires

Palavras-chave

Bicicleta Mobilidades Etnografia Buenos Aires 
environments is re-elaborated. Finally, I identified the conformation of a socialisation space through the intentional and collective tours that are emerging in the city and which add greater heterogeneity to urban cycling.

\section{Introducción}

Distintas ciudades del mundo están realizando modificaciones en sus formas y flujos urbanos a partir del fomento y necesidad de la movilidad sustentable. Una de las propuestas que está teniendo mayor aliento es la del ciclismo urbano, sus ventajas se dirigen hacia la gestión de los servicios públicos y para enfrentar problemas relacionados al transporte, además ofrece beneficios psicológicos (Caballero et al., 2014) y en la salud física (Lorenc et al., 2008). En la ciudad de Buenos Aires pueden observarse algunos impactos de la práctica del ciclismo urbano, por mencionar dos ejemplos, la infraestructura exclusiva para el flujo de las bicicletas como ciclovías y bicisendas, y el servicio del Sistema de Transporte Público en Bicicleta. Precisamente estas intervenciones propiciaron que en el 2015 el "Copenhagenize Index" incluyera esta ciudad en el lugar catorce dentro de las veinte mejor valoradas a nivel mundial por los esfuerzos para fomentar el uso de la bicicleta como medio de transporte. Así, un perfil general de Buenos Aires aparenta un ambiente ideal para el ciclismo.

Sin embargo, la práctica del ciclismo urbano es mucho más heterogénea que aquella ofrecida por la imagen de una persona sonriente en bicicleta, va más allá de hacer girar las ruedas que permiten el desplazamiento. Es una práctica individual que requiere de habilidades y conocimientos específicos, en algunas ocasiones se acompaña de un posicionamiento político y, al mismo tiempo, es una práctica colectiva retroalimentada de las experiencias en ambas dimensiones. Así, el ciclismo urbano es una práctica específica de viaje por la ciudad y con ello también conforma maneras de relacionarse con los entornos urbanos. Un ejemplo del dinamismo y posibilidades que abre esta práctica es la emergencia de paseos colectivos que se están realizando en Buenos Aires y que ofrecen otro tipo de interacciones sociales.

Por otro lado, esta práctica se enmarca en las características y dinámicas específicas de cada ciudad, influye y es afectada por múltiples aspectos sociales, culturales, económicos y políticos (Horton, Rosen y Cox, 2007; Spinney, 2009; Vivanco, 2013). Generalmente se convive entre políticas públicas que incentivan la elección por la bicicleta junto con un ambiente que puede llegar a ser hostil por las condiciones de la infraestructura ciclista y por la violencia vial que puede percibirse al transitar por la calle.

En medio de estos entramados de posibilidades es que la bicicleta (re)aparece en años recientes con mayor visibilidad, precisamente por el cruce de distintos procesos urbanos vinculados de manera directa (demanda de servicios por algunos sectores de la población, políticas de movilidad sustentable) o indirecta (turismo, gentrificación, clústeres creativos, etc.). En conjunto generan un efecto que se observa a nivel global, un auge del ciclismo urbano tanto en la práctica como en interés por parte de la academia. Así, el trabajo aquí expuesto surge de la inquietud por comprender las interacciones y características que engloba la práctica del ciclismo urbano en la ciudad de Buenos Aires, y capturar un momento específico de los procesos cambiantes de las prácticas de movilidad urbana.

\section{Perspectivas del ciclismo urbano}

Algunas investigaciones que han revisado antecedentes de la bicicleta (Herlihy, 2004; Penn, 2011; Vivanco, 2013) mencionan que las publicaciones de finales del siglo XIX 
y principios del siglo XX eran, básicamente, de tipo reivindicativo o declaratorias a favor de la libertad de movimiento que ofreció este medio de transporte (de manera especial para las mujeres, Penn, 2011). Con el crecimiento de las urbes y su correspondiente diversificación de problemas y necesidades, junto con las ideas de progreso y el contexto global de economía neoliberal, se fueron perfilando indagaciones hacia la movilidad urbana. Éstas se inclinaron hacia los medios de transporte que ofrecieran más velocidad para conectar puntos con rapidez, mayor alcance medible en kilómetros $\mathrm{y}$, de manera prioritaria, que permitieran trasladar a una gran cantidad de personas. El sistema de transporte subterráneo de Buenos Aires, el único metro de la Argentina, es un ejemplo de la materialización de las ideas de modernidad y progreso (Zunino Singh, 2014). En esta dinámica el automóvil ha sido el medio predominante, lo que se ha transferido a las representaciones e infraestructura urbana (Edensor, 2004; Hall, 2003) y ha opacado otras posibilidades de transporte.

Luis A. Vivanco (2013) menciona que observar la bicicleta en los momentos actuales es un ejercicio de re-observación hacia un objeto viejo como si fuera uno nuevo. Lo es por el funcionamiento básico de este objeto (Whitt y Wilson, 1995), al mismo tiempo que ha experimentado modificaciones en sus componentes, en las formas, y en la diversificación de la práctica del ciclismo. Aunque, Vivanco también advierte que la bicicleta ha tenido y tiene incremento de uso en interrelación con los cambios sociales e históricos según las ciudades que observemos (Vivanco, 2013:XX). En este sentido, lo que sucede en el momento de observación y registro de una investigación es casi una fotografía que corresponde a un periodo y una dinámica especifica.

En la introducción de Cycling and Society, Horton, Cox y Rosen (2007:8-9) señalan que hay cuatro perspectivas de indagación en torno a la bicicleta y al ciclismo. Una es histórica, con interés en los cambios de la manufactura de la bicicleta y sus impactos sociales en diferentes momentos. La segunda hacia el ciclismo deportivo al observar las variantes deportivas y competiciones. La tercera incluye la ingeniería, diseño y planeación para incrementar el uso de la bicicleta como un medio de transporte. La cuarta perspectiva se centra en los efectos positivos del ciclismo en la salud, así como de análisis de accidentes (Horton, Cox y Rosen, 2007:8-9). También indicaban que había otras tres perspectivas iniciales al momento que realizaron la revisión, una vinculada a la sociología de la ciencia y tecnología; otra desde la geografía social y cultural que se centraba en la experiencia del ciclismo; y la tercera que se vinculaba a las políticas públicas en torno a la bicicleta y el ciclismo (Horton, Cox y Rosen, 2007:9). Como puede observarse es un espectro muy acotado que corresponde a un momento histórico y que se ha ampliado a partir de otras investigaciones y estudios.

\section{Agenda pública y sociedad}

Una de las perspectivas que se ha desarrollado en el ciclismo urbano es la que proviene de agentes regionales e internacionales que se enfocan en políticas públicas. Un aspecto favorable de este tipo de documentos es que van disgregando diferentes elementos a considerar en la planificación urbana para mayores usos e impactos de la bicicleta. Un ejemplo es la guía del Banco Interamericano de Desarrollo (2015), en la que destacan 24 indicadores divididos en factores relacionados al ciclismo y sus impactos, además exponen algunos ejemplos de buenas intervenciones (como la intermodalidad en el trasporte público y los servicios de bicicletas públicas). Por su parte, la Secretaría de Transporte de la ciudad de Buenos Aires difunde un Manual del ciclista (2017) en la página web del gobierno local, que puede considerarse la versión oficial y normalizada de utilizar la bicicleta como medio de transporte.

Desde otra posición, la empresa Copenhagenize Design (2013) realizó un estudio antropológico con la intensión de apoyar el diseño de políticas públicas, The Bicycle 
Choreagraphy of an Urban Intersection, en el cual un equipo interdisciplinario observó el comportamiento de ciclistas para identificar patrones y movimientos, es decir, las líneas de deseo que conforman una coreografía urbana de la movilidad. Un antecedente de estas indagaciones lo observamos en Parkin, Ryley y Jones (2007), quienes indican que los análisis cuantitativos requieren de metodologías cualitativas para complementarse, por ejemplo, para diferenciar entre indicadores que predominan en los estudios de transporte como tiempo y distancia, hacia aspectos más subjetivos que influyen en las elecciones y opciones de los trayectos de ciclistas.

Siguiendo la línea de propuestas que buscan impactar las relaciones entre los actores que comparten flujos y espacio en la ciudad están todas aquellas que provienen de las organizaciones civiles y de colectivos. Cabe advertir que no se trata de estudios sino de recursos educativos para el tránsito urbano. Una de las primeras propuestas es la Guía del ciclista urbano realizada por la Asociación de Ciclistas Urbanos y En Bici Seguro (2013), en donde se sintetiza el reglamento de tránsito que afecta a los ciclistas y además ofrecen algunos consejos prácticos para circular por la ciudad. Por otra parte, hay toda una serie de materiales educativos disponibles en la web que se enfocan en la convivencia entre conductores, ciclistas y peatones, entre otras propuestas, por ejemplo, utilizar la bicicleta como recurso didáctico en las escuelas o utilizarla para ir al colegio.

\section{Diferencias e identidades}

Algunos acercamientos al ciclismo ponen mayor énfasis en las subjetividades de las personas que practican el ciclismo. Es el caso de la investigación de Aldred (2013), quien se enfocó en las identidades sociales al considerar que las calles no sólo son espacio de tránsito sino también arenas de formación de identidades en las que se ponen en juego diferenciaciones sociales (Aldred, 2013:253-254). Las identidades se conforman, entre otros aspectos, por las actividades que realizamos y las percepciones que tenemos de nosotros en relación a las interacciones con las demás personas. Así, Aldred ofrece dos tipos de representaciones, el buen ciclista y el ciclista desatinado (2013:259-264). Precisamente, ambas encajan con las asignaciones que los ciclistas porteños se hacen entre sí y con las negociaciones de valores vinculados a la práctica del ciclismo, con ello conforman representaciones del buen ciclista urbano y de otros tipos de ciclismos desde los cuales se sitúan unos y otros.

Por otra parte, Lugo (2012 y 2013) analiza la práctica del ciclismo urbano en relación con las diferencias de género y de etnicidad. Así, la práctica del ciclismo es atravesada y se realiza a partir de otras relaciones basadas en las diferencias y desigualdades, lo que genera que en torno al ciclismo se formen diversos grupos en respuesta a las identificaciones que les convocan, según sus experiencias y contextos estructurales.

Lugo mantiene una estrecha relación con la etnografía porque le permite involucrar de manera más participativa a quienes disfrutan y sufren la bicicleta. Esto último le permite indicar que las metodologías cualitativas son necesarias para incidir en la planificación de la infraestructura ciclista. En la línea del activismo con una visión crítica de la equidad del uso del espacio público, la edición de Bicycle Justice and Urban Transformation (Golub et al., 2016) ofrece estudios de caso en los cuales la bicicleta sirve de articulación de diversas relaciones sociales, Lugo y otros académicos/activistas desmontan la imagen estereotipada del ciclista (acomodado, educado, clase media, etc.) y reconocen diversas manifestaciones por las cuales deberían pasar las intervenciones urbanas.

\section{Movilidades y ciclismo}

Sheller y Urry (2006) indican que el paradigma de las movilidades influye en las formas de observar las prácticas que implican los desplazamientos de personas, objetos e ideas. 
Así, uno de los ámbitos que atienden las movilidades se dirige hacia la realización de múltiples prácticas corporales que implican el movimiento, sus representaciones y los diversos significados que se van configurando de y en las prácticas de movilidad. Precisamente, una de las perspectivas emergentes que lograron captar Horton, Cox y Rosen (2007) es la que se enfoca en las experiencias de los ciclistas.

Las investigaciones que pueden enmarcarse en esta perspectiva son aquellas que buscan identificar las diferentes contradicciones entre prácticas y sus significados. Un trabajo que ejemplifica esta orientación es el realizado por Švarc (2017), en el cual ofrece una detallada descripción de diferentes prácticas de ciclismo urbano a partir de seguir y acompañar a cuatro personas que participaron en su trabajo. La muestra pareciera pequeña, pero, la cualidad de las descripciones le permiten realizar amplias comparaciones y contraposiciones.

Un ejemplo distinto de este tipo de acercamientos es el de Van Duppen y Spierings (2013), quienes se enfocaron en las interacciones desde la experiencia del ciclismo con el entorno urbano, y con lo cual identifican divisiones y conexiones entre los diferentes territorios que se encuentran en el continuo urbano de la ciudad. Relacionado a la experiencia ciclista y lo urbano, Latham y Wood (2015) indagan la relación de condicionantes externos, específicamente las infraestructuras urbanas, en el trazado y las adaptaciones que los ciclistas realizan sobre sus trayectos para trasladarse por la ciudad. Por su parte, Simpson (2016) realiza una exploración más sensorial al concentrarse en las atmósferas y los ambientes que perciben los ciclistas a su paso por las infraestructuras instaladas para el ciclismo urbano.

Por otro lado, Larsen (2016) recalca que el ciclismo es contradictorio porque las personas le asignan distintos significados, los cuales provienen de sus experiencias individuales y de la conformación del ciclismo de manera colectiva. Los significados hacia la bicicleta y al ciclismo siempre están flotando y modificándose, incluso coexisten sentidos contradictorios. Por ejemplo, la posibilidad de moverse rápido puede ser vista como una posición de poder y privilegio (Aldred, 2015), sin embargo, la velocidad depende de que sea asignada a la bicicleta o al automóvil, o en relación a las posiciones sociales. Así, el ciclismo está inmerso en otros conjuntos de relaciones que influyen en las experiencias individuales y colectivas, como las relaciones de clase (Lugo, 2012), o puede llegar a ser una cuestión de vida o muerte según existan o no hábitos de respeto hacia los demás actores viales (Švarc, 2017).

Ante este abanico de perspectivas, la invitación a pensar el ciclismo de maneras distintas que realizan Horton, Cox y Rosen (2007) en Cycling and Society continúa vigente. Así, en este artículo se retoma el paradigma de las movilidades para realizar una lectura a los procesos vinculados al ciclismo, los cuales fluyen entre las experiencias individuales y colectivas, y que están en transición.

\section{Metodología}

El acercamiento que realicé al ciclismo urbano en la ciudad de Bueno Aires fue en el marco de una investigación cualitativa enfocada hacia las prácticas de movilidad activa en contextos urbanos. El trabajo de campo consistió en un periodo de ocho meses, de noviembre del 2016 a junio del 2017. Al seguir los parámetros del método etnográfico la observación participante me llevó a relacionarme con personas ciclistas y participar en las actividades que se realizan en la ciudad en torno a distintos tipos de recorridos en bicicleta. De manera paralela realicé entrevistas semiestructuradas con participantes de los paseos organizados por diferentes agrupaciones y colectivos, con miembros de organizaciones vinculadas con el ciclismo urbano, y con actores clave o representativos de la práctica del ciclismo. 
Por las características de la investigación he optado por recuperar algunos elementos de la autoetnografía (Ellis y Bochner, 2000) como guía para el registro de la información y para elaborar un relato que exponga experiencias y situaciones del ciclismo urbano. Esta decisión metodológica surgió al identificar que iba manteniendo específicas interacciones con las personas, realizaba lo que éstas hacían, lo que me permitió contrastar la información que me iban proporcionando con mis propias vivencias (disfrutar y sufrir la bicicleta). Así, mi presencia durante los paseos en bicicleta fue necesariamente encarnada (Csordas, 1994), y con ello compartida y contrastada con las demás personas (Lugo, Mattheis y Aguirre, 2017). Bajo esta premisa intenté relacionar la habilidad de utilizar la bicicleta, aparentemente sencilla, con las dinámicas que se van dando al compartir el espacio público desde y en relación con la práctica del ciclismo. Este propósito se benefició de las reflexiones de ciclistas desde sus experiencias individuales y colectivas, y porque quien investiga tiene referencias desde su propia experiencia. Sin embargo, cabe aclarar que en este artículo la autoetnografía sirve de guía pero no se expone como un texto.

Esta manera de proceder en torno al ciclismo tiene sus antecedentes en los estudios del transporte y de la movilidad urbana. Un acercamiento previo es el de Phil Jones (2005), quien fue registrando su propia práctica durante sus viajes al trabajo, y si bien no indicó que se trata de una autoetnografía sí comparó las maneras de entender la ciudad, una de ellas desde la experiencia ciclista. Otro trabajo es el de Alexandra Coghlan (2012), que utiliza el recurso de la autoetnografía para explorar la práctica ciclista desde la experiencia personal, aunque en este caso del ciclismo de turismo y de eventos deportivos.

De manera más perfilada, Larsen (2014) indica que la autoetnografía le permitió exponer diferentes cualidades corporales que implica el movimiento. En su trabajo despliega algunos señalamientos en torno a la adaptación de hábitos y rutinas a partir de su propia experiencia al modificar las situaciones en donde realizó el ciclismo, una fue en un lugar conocido, otra al adaptarse a un lugar nuevo, y la tercera se trató de una manera diferente de realizar el ciclismo (Larsen, 2014:64-69). En una línea más crítica Adonia Lugo, Allison Mattheis y Maryann Aguirre (2017) también utilizaron la autoetnografía para el estudio de la bicicleta en la ciudad, exploraron este tipo de narrativa para exponer las perspectivas desde las cuales sus experiencias estaban marcadas por el género, la raza y la etnicidad.

Por otro lado, Herlihy (2004) indica que difícilmente se podría encontrar alguna mención a la bicicleta sin que esté relacionada con vivencias propias y que se transcriban como anécdotas, esta afirmación es secundada por Augé al indicar que la bicicleta está incorporada a las historias personales (2009). Precisamente, durante el trabajo de campo fui encontrando una serie de blogs, páginas y servicios ligados a internet, dedicados al ciclismo urbano en donde se iban registrando y relatando vivencias articuladas a la bicicleta. Las descripciones estaban inmersas en historias personales con lo cual se hacían mucho más accesibles al contrastarlas con las experiencias propias. Otro tipo de información se relacionaba a la organización de recorridos colectivos o a grupos de ciclistas. Así, una parte del trabajo de campo la destiné para transitar por el espacio digital, además de recuperar información relacionada al ciclismo para enterarme de las actividades y paseos colectivos.

\section{A rodar la rueda}

Es generalizado diferenciar entre saber andar en bicicleta que realizar ciclismo urbano, pues implica muchas más cosas que simplemente mantener el equilibrio. En este sentido, debo indicar que yo me hice ciclista urbano en Buenos Aires, durante los ocho 
meses que duró el trabajo de campo fui cambiando de habilidades y fui acumulando conocimientos relacionados a la bicicleta, al tránsito y los reglamentos, y fui ampliando el reconocimiento del entorno urbano que conforma la ciudad. Este proceso individual lo conecté con las experiencias que me fueron compartiendo las personas con las que fui interactuando.

La mayoría de las personas ciclistas me indicaron que habían aprendido a andar en bicicleta desde la infancia, para algunas estaba directamente conectada con esa etapa de sus vidas y años después la habían retomado. Otras personas comentaron que les había acompañado durante varias etapas de manera intermitente. Unas pocas personas comentaron que habían tenido alguna vivencia desagradable o que nunca habían logrado desarrollar la habilidad para mantener el equilibrio, así que hasta años recientes comenzaron a transportarse en bicicleta. La manera inicial como nos acercamos a la bicicleta influye en las relaciones que establecemos, en las percepciones que tenemos del entorno urbano, y de las posibilidades que se pueden generar al utilizarla como medio de transporte. Sin embargo, hay un elemento que se comparte, la conformación de una autoidentificación como ciclista urbana/o que se basa en las experiencias corporales. Como dice Mauss (1950), las vivencias, que corresponden a diversos usos del cuerpo, se articulan socialmente haciendo que el cuerpo sea la herramienta desde la cual se capta y se comparte el mundo.

Hay dos maneras básicas para comenzar con el ciclismo urbano, en solitario al salir con la bicicleta hacia algún punto de la ciudad, o en colectivo al buscar algún grupo que realice paseos en bicicleta. Entre estos dos extremos hay varias posibilidades como salir en pareja o en pequeños grupos de amistades y en familia. En mi caso, y al igual que les sucedió a varios de los ciclistas de Buenos Aires, el comienzo fue en solitario, fundamentalmente porque se asume que es suficiente con mantener el equilibrio en la bicicleta, sin embargo, en el transcurso del primer tramo uno va reconociendo que no es lo mismo saber andar en bicicleta que hacer ciclismo urbano. Es una obviedad que sólo se puede comprender a partir de realizar el ejercicio como un actor más en el flujo del tránsito, de registrar en carne propia que es una interacción tensa.

La segunda manera es buscar algún grupo con el cual iniciar los primeros paseos. La difusión de boca a boca es útil (como sigue siendo con la Masa Crítica Bs. As.) pero es mayor la circulación de información por medios digitales. Así, basta con encontrar el dato adecuado, leer bien las condiciones (por ejemplo, el uso de casco obligatorio en algunos grupos), solicitar más detalles y acudir. Los medios más eficaces son las redes sociales, los blogs, y páginas web, donde se puede encontrar información general de las bicicletas, de los paseos colectivos, de bicicleterías, de las leyes de tránsito, y recursos para interactuar entre usuarios y organizar recorridos, como MeetUp, la sección Recorridos de La Guía Biker o el hilo de Salidas en Bici de la página AmigosBuenosAires, entre muchas otras.

Durante los primeros días del trabajo de campo estuve siguiendo las publicaciones de un grupo de Facebook, Masa Crítica Bs. As., porque quería salir en el paseo que harían a mediados de noviembre con la luna llena. Me sirvió para ponerme al día de los comentarios que se realizaban en aquel foro virtual, además fui descubriendo otras páginas de diferentes grupos que también realizaban paseos. Algunos solicitan mayores requisitos, por ejemplo, el uso obligatorio del casco, la mayoría de edad para poder asistir, o advertencias sobre complementos de repuesto (parches, bomba de aire, etc.). Por otro lado, esta inmersión virtual me sirvió para ir identificando que no todo circulaba necesariamente por la red social de Facebook, sino que había otros sitios en donde también se gestionan paseos y que eran igual de importantes porque congregaban a muchas personas, al menos virtualmente. Algunos sitios clásicos son Amigos del Pedal o La Guía Biker. 
Con los primeros viajes y paseos se va aprendiendo que las condiciones y la infraestructura pueden ser favorables para el ciclismo. Hay carriles exclusivos para la bicicleta, hay campañas y un departamento del gobierno local dedicado a la movilidad sustentable, un sistema de transporte público en bicicleta gratuito, y mucha gente desplazándose en bici. Pero, las habilidades de uno pueden estar lejos de encontrarse al nivel adecuado. Con el paso del tiempo y en cuanto se van adquiriendo aquellas habilidades mínimas para administrar el movimiento, lo que se hace reconocible es que las condiciones son adversas. Hay sesgos en la planeación y el diseño urbano, escaso mantenimiento a la infraestructura, mínimas campañas para cambios de hábitos viales dirigidas a conductores de vehículos motorizados, entre otros. Entre mayor habilidad para circular en bici también cambian las percepciones del tránsito en la ciudad.

Por mencionar algunos datos de la violencia vial en la ciudad de Buenos Aires la Defensoría del Pueblo indica que durante el 2016 se registraron 9.090 siniestros viales que dieron como resultado a 9.842 víctimas, de las cuales 66 fueron fatales en el sitio del incidente (DPCABA, 2017:6). Ese mismo año las víctimas ciclistas dieron un total de 591 (DPCABA, 2017:10). En febrero del 2017, el colectivo de organizaciones civiles Argentina en Bici (ABi) publicó un comunicado en el que informaba que durante enero y febrero se habían registrado 19 muertes ciclistas en todo el país. Durante enero del 2018 también se realizaron manifestaciones de reclamo por mayor seguridad vial. En este sentido, muchos comentarios co-presenciales durante los paseos y también virtuales en los foros y en las redes sociales, advertían un ambiente de riesgo para transitar en bicicleta.

Aun así, se encarna el descubrimiento de que transitar en bicicleta es una experiencia placentera. A pesar de que dicha cualidad difiere, como advierten Horton, Cox y Rosen (2007), cuando se trata de una opción que cuando es un recurso condicionado por las circunstancias, y esto también lo manifestaron los ciclistas al decir que es un medio de transporte más sencillo, les da libertad de desplazamiento, es más barato, y es rápido. Quizá el punto de coincidencia es que al inicio sólo hay que convertir el ciclismo en un hábito.

\section{Transitar por la ciudad}

Hay un conocimiento tácito que tienen las personas que han vivido por muchos años en la misma ciudad, aunque muchas no se den cuenta de que lo poseen pero que sobresale cuando tienen que ubicar lugares, sitios, calles, o de la fama y estereotipos que tienen algunos barrios. Es un conocimiento acumulado desde el cual se disponen a transitar y que, al mismo tiempo, se va reelaborando en cada ocasión. Es en las conversaciones cuando, por ejemplo, al dar indicaciones de algunos lugares las personas se abstraen para hacer un ejercicio de reconocimiento de los fragmentos de la ciudad que han recorrido y conocen. Algunas ocasiones esos conocimientos se ven contrastados con los de otras personas que han tenido otras experiencias, $o$ porque utilizan más un medio de transporte que otro. Por ejemplo, Lauriano, un ciclista que coordina un grupo de recorridos urbanos en bicicleta, me comentaba que puede trazar distintas rutas de la ciudad mientras está en la bicicleta, pero, una vez caminando le cuesta orientarse.

También, las rutinas cotidianas han dotado de dinámicas a la ciudad, por lo que son distintas las actividades que se realizan en las mañanas y en las noches, o las que se realizan los fines de semana. En conjunto modifican nuestras percepciones y experiencias de la ciudad. En este sentido, cada desplazamiento ofrece posibilidades de ampliar los conocimientos que se tienen del entorno. Quizá algunas personas no se den cuenta de 
que ocurre esta ampliación, pero quienes sí realizan un ejercicio de reflexión reconocen que han adquirido otras perspectivas sobre aquellos entornos urbanos que conocían y que en contraste ahora pueden observar otros matices.

Uno de los aspectos importantes que observé durante el trabajo de campo es que los grupos cercanos son fundamentales para incentivar la práctica del ciclismo urbano. La familia, el grupo primario, juega un papel básico en el uso de los diversos medios de transporte, entre ellos el uso de la bicicleta porque, si bien la decisión es individual, también tiene una parte colectiva que es precisamente la transmisión de una práctica (Larsen, 2016; Turner y Bruner, 1986). Uno de los ejemplos más contundentes es el de Riky, un ciclista que recuperó la salud con esta práctica y por ello se ha encargado de trasladar su pasión por el ciclismo urbano hacia los integrantes de su familia. Los siguientes grupos de influencia suelen ser las amistades, sin embargo, lo que encontré es que hay quienes se sienten acompañados por un grupo de amigos para realizar paseos, pero, también hay personas que buscan otros grupos porque sus amistades no utilizan la bicicleta. Este hecho refuerza la generalidad de que el uso de la bicicleta es una decisión individual, aunque apoyada por percepciones de acompañamiento y de las propias habilidades (Caballero et al., 2014).

Por otro lado, al transitar por la ciudad se desarrollan algunas habilidades, unas se escuchan constantemente en las pláticas, pero, hasta que uno se encuentra en esa situación es que se pueden apreciar. Esto no quiere decir que lo que se comparte en charlas sea descartable, sino al contrario, porque se ha escuchado es que se puede reconocer cuando sucede. En específico las personas me fueron ayudando a desarrollar dos habilidades (no son las únicas ni las más importantes sólo las que fueron resaltando más durante el trabajo de campo): ser predecible y leer las acciones de otras personas.

Ser predecible significa pre-visualizar mis movimientos. Al terminar un paseo en Devoto le pregunté a una chica del grupo si sabía cómo llegar a una ciclovía, Laura me dijo que debería seguir por la Av. del Carril (que después es La Pampa) hasta llegar a la calle Superí donde hay una ciclovía. Me dijo que me acompañaría porque ella seguiría un poco más para continuar con la ruta que ya había planificado. Íbamos en paralelo por la calzada y un coche pasó cerca de nosotros, entonces Laura comentó que en una ocasión iba con alguien de la misma manera y al querer dar una vuelta hacia la izquierda casi les ocurre un accidente con el coche que venía atrás de ellos. Así, me aconsejó que en las futuras salidas no debía dar por hecho que quien va atrás de mí sabe lo que yo realizaré, ya sea frenar, girar, acelerar, o lo que sea, que debía pre-visualizar mis movimientos para indicar con señales (de mano o con el lenguaje corporal) lo que haría, con esa previsión me ahorraría un susto. Por supuesto, me advirtió Laura, eso no me hacía inmune ante algún imprudente en coche. Este tipo de recomendación se repitió en otras ocasiones, con otras personas quienes evocaron las situaciones que habían vivido.

Leer acciones de otras personas. En un recorrido con Ciclofamilia (agrupación que realiza eventuales capacitaciones para personas interesadas en el ciclismo urbano), Fernando, el instructor, me indicó que una manera de circular con seguridad en la bicicleta es mantener la atención en el trayecto que uno va realizando, lo que implica observar las condiciones de la calzada, de los carriles de bicicleta, y apegarse en lo posible el reglamento de tránsito. Además, observar el entorno y las acciones de las personas, tanto ciclistas, peatones y conductores de vehículos motorizados (coches y motocicletas). Pero, a pesar de las precauciones, lo mejor es esperar lo inesperado, por ejemplo, una persona que cruza la calle por el medio saliendo entre algunos coches, o que una persona abra la puerta de un coche sin verificar los laterales, o que sea un ciclista sin experiencia que se arroja, entre otros casos. Así, uno debe realizar una lectura constante de lo que va aconteciendo en nuestro alrededor mientras se circula en la bicicleta. Fernando me indicó que con el tiempo se hace un hábito, una manera de hacer y de transitar. 
Unos días después de la capacitación estuve observando durante varios días a los ciclistas para identificar sus maneras de leer el contexto del tránsito y el entorno. Pude comprobar que efectivamente mantenían una interacción con lo que iba ocurriendo, por ejemplo, al regular sus velocidades con los frenos y el pedaleo, los giros de cabeza para observar alrededor y atrás, el zigzagueo ante los obstáculos (incluso en las ciclovías) y los coches. Con el paso del tiempo, también reconocí esas habilidades en mi propio desplazamiento para mantener un ritmo y una prevención en mi manera de circular, la cual no se correspondía necesariamente con las maneras como lo realizaban los otros ciclistas, sino que era una manera particular que había desarrollado a partir de mis propias experiencias y percepciones.

Con las posteriores observaciones durante el trabajo de campo reconocí que no hay una sola manera de andar en bicicleta por la ciudad, sino que cada persona va desarrollando su propia manera de transitar a partir de las habilidades que cada quien va desarrollando (aunque siempre en relación a otros ciclistas). A veces, este mismo detalle causa conflicto entre los ciclistas porque algunas personas consideran que hay quienes circulan mal, que se ponen en riesgo o dan una mala imagen del ciclismo. Sin embargo, una vez cumpliendo los lineamientos necesarios de seguridad vial (ACU y En Bici Seguro, 2013; Secretaría de Transporte, 2017) el ciclismo urbano es en sí una práctica heterogénea.

\section{Desde el cuerpo}

Las prácticas de movilidad (activa) son necesariamente prácticas corporales. Las percepciones de cada quien sobre las habilidades propias y del entorno se relacionan y reelaboran mutuamente durante cada trayecto desde las vivencias del cuerpo, al mismo tiempo, se realizan con base en las experiencias previas. Así, cuando vemos a un ciclista transitando con destreza en las calles estamos viendo, aunque no aparente serlo, un cúmulo de aprendizajes e interacciones múltiples. Durante esos momentos intervienen los sentidos y la disciplina física (Larsen, 2014), además de las estructuras urbanas por donde se transita (Latham y Wood, 2015).

Mario Jordi Sánchez, al retomar la noción de técnicas corporales de Marcel Mauss, indica que las maneras de transitar se expresan por "el proceso de aprendizaje colectivo y de evolución-cambio de dichas técnicas" (Jordi Sánchez, 2016:219), en este caso de aquellas vinculadas a la práctica del ciclismo urbano. Por ello, Jordi Sánchez indica que pueden observarse particularidades de la cultura ciclista de cada ciudad a partir de observar la incorporación de diversos detalles que aparentan sencillez aunque más bien corresponden a un complejo entramado de conocimientos, habilidades, movimientos, gestos, entre otros:

La lista es interminable: la altura a la que se coloca el sillín (más bajo de lo normal da seguridad a los principiantes), las dudas a la hora de cruzar un paso de peatones, la inmediatez con la que se emprende la marcha, la determinación con la que se afronta una situación de peligro, la firmeza al sujetar el manillar, la oportunidad en el cambio de marchas (que se nota en la cadencia adecuada del pedaleo), la velocidad con la que se afrontan las curvas, la posibilidad de "bailar" sobre los pedales, emulando a los "escaladores" ciclistas para subir las cuestas, la decisión con la que se negocian las bajadas, un mayor acople a la máquina, un estilo más natural, armónico y coordinado, una expresión corporal relajada, esa sensación de que no cuesta trabajo circular en bicicleta por la ciudad, siendo tan difícil... (Jordi Sánchez, 2016:219).

La lista es amplia. Además, Mauss (1950) sugiere que las técnicas corporales no solo cambian de un sitio a otro sino también a través del tiempo, por lo que se podría decir 
que las maneras como se realizaba el ciclismo unas décadas atrás en la ciudad de Buenos Aires han cambiado (visible muchas veces en las fotografías antiguas), aunque son antecedentes de las prácticas actuales. Las tecnologías también han cambiado recíprocamente con las técnicas corporales, se han adaptado o se han reciclado. Ahora conocemos otros detalles en relación a las medidas y tallas, los materiales, la mecánica, y todas aquellas opciones vinculadas a la elección de la bicicleta y sus componentes. Actualmente hay una variedad de bicicletas acopladas al cuerpo y a las actividades: de ciudad, de carrera, plegable, de montaña, híbrida (Secretaría de Transporte, 2017:34), hay de otras variedades como triciclo de carga, fixie, o tuneadas (unas para trabajar y otras para lucir), algunas explicitan una relación complementaria entre objeto y medio de transporte (Vivanco, 2013:23-25). Las posibilidades se amplían y las técnicas corporales se van innovando.

En este sentido, Mariana, una ciclista y mecánica de bicis, me comentó que al utilizar este medio de trasporte hay toda una predisposición y adaptación entre el cuerpo y la bicicleta. Por ejemplo, calibrar los componentes de acuerdo a nuestro cuerpo para evitar alguna molestia física, así como llegar a reconocer fricciones, ruidos, entre otros detalles técnicos que se sienten al viajar en bici, necesarios para circular bien y para mantener la bicicleta en buen estado. Así, la bicicleta se convierte en una extensión del cuerpo que nos permite trasladarnos de una manera distinta del caminar o del automóvil. Además, hay un asunto de sensaciones que se perciben al pedalear y que deben cuidarse porque las averías o un ajuste erróneo puede repercutir en malestares físicos. Precisamente, al utilizar una bicicleta del Sistema de Transporte Público en Bicicletas se puede reconocer un acoplamiento forzado de nuestros cuerpos. Mientras que las bicicletas propias están adaptadas, son cómodas, las hemos ajustado y podemos reconocer nuestros movimientos con facilidad.

\section{Mujeres ciclistas}

En una tarde de la última semana de febrero estuve platicando con un ciclista que ha realizado, junto con un equipo de investigación, algunos estudios vinculados a la movilidad sustentable. Paul me comentó algunos aspectos que las personas toman en cuenta al elegir la bicicleta como medio de transporte, uno de esos aspectos fue la percepción de las propias habilidades, la cual era baja en las mujeres que han participado en sus estudios. Un ejemplo que dio es el de una chica quien les dijo que ella circulaba por las ciclovías y bicisendas pero cuando éstas terminaban se bajaba y continuaba caminando hasta su destino. Aunque, al mismo tiempo, Paul mencionó que la elección de la bicicleta por parte de las mujeres hacía referencia a un sentido de movilidad libre. Lo que marcaba la distancia entre ambos aspectos era la sensación de seguridad al circular en las calzadas con dos condicionantes, uno externo, que refiere a la velocidad y violencia vial que se percibe al transitar (Wright, 2013), y otro individual, que es el ejercicio de habilidades para circular con destreza.

Un mes antes, a finales de enero del 2017, una chica publicó una experiencia de acoso callejero mientras transitaba en bicicleta. Ella describió que al terminar una reunión subió a su bici, circulaba tranquila aprovechando que había poco tráfico por ser de noche, pero, en un cruce mientras esperaba el verde para continuar se acercó un taxi y el chofer la tocó, ella le gritó y el taxi se fue. Ella relata que fueron unos segundos que la hicieron sentir vulnerable, y a pesar de que el acoso en general es un tema recurrente entre amigas y conocidas por la frecuencia con la que ocurre, ella no esperaba que le sucediera y menos tener aquella sensación de inseguridad. Sin embargo, también relató que frente aquella situación, sale a las calles con la bici porque le sigue dando una sensación de empoderamiento por la libre movilidad y, ahora, por una demanda de lucha contra el machismo. El relato lo llamó: "La calle es nuestra". 
Llegó el 8 de marzo, día de la mujer, con una multitudinaria marcha que se sumó a un paro internacional de mujeres, en donde se realizaron manifestaciones en contra de las diversas desigualdades de género. El paro internacional tuvo distintas demandas específicas en cada país y ciudades. En el caso de Argentina uno de los reclamos más extendidos fue "fin a la violencia machista", ya que durante los meses anteriores se habían presentado varios casos de feminicidio. En torno a este evento se organizaron un grupo de mujeres que decidieron realizar un paseo entre mujeres, desde el cual reivindican "el derecho de las mujeres a circular libremente por el espacio público y hacer escuchar su voz".

Mujeres sin Frenos es un grupo de mujeres que utilizan la bicicleta para circular por la ciudad. Algunas de sus integrantes reconocen que las maneras como se mueven hombres y mujeres por la ciudad son distintas, las cuales están vinculadas a las diferencias de género que influyen a su vez en la conformación de desigualdades en base a esas mismas diferencias. Así, consideran que el uso de la bicicleta es una herramienta emancipadora porque es un medio accesible, les brinda libre movilidad, mayor sensación de seguridad (que caminando y en relación al acoso callejero), independencia para trasladarse (este punto ha sido señalado por todos los ciclistas urbanos, pero, tiene mayores repercusiones para las mujeres), entre otros aspectos. Durante cualquier recorrido por la ciudad es evidente que hay amplia cantidad de mujeres ciclistas, pero, continúan siendo más los hombres. Por ello, este grupo intenta hacer visible el uso de la bici por parte de las mujeres en el espacio público, al mismo tiempo que generar un espacio de mujeres para mujeres.

Entre 1895 y 1897 se publicaron tres manuales para mujeres, el primero fue "A Wheel within a Wheel" (1895), el segundo "Bicycling for ladies" (1896), y el tercero "Lady Cycling" (1897), en los cuales se daban consejos para las mujeres que querían utilizar la bicicleta. Del primero, Mackintosh y Norcliffe (2007) indican que la autora era una educadora, quien pensaba que el problema no era que las mujeres utilizaran la bicicleta sino el cómo se utilizaba, por ello su interés en ofrecer un manual. En el segundo se dan recomendaciones generales, sólo en el capítulo 8 se menciona que las mujeres pueden utilizar las herramientas para arreglar la bicicleta. En el tercero, la misma autora indica que está escrito por parte de una mujer ciclista para mujeres ciclistas, algunos de los consejos se dirigen a la vestimenta, la alimentación y hacia los modales. Resalta que Erskine (1897) menciona que preguntarse si las mujeres deberían o no practicar el ciclismo era, ya en su momento, una pregunta ociosa porque las mujeres estaban utilizando la bicicleta. Huerta y Gálvez (2016) señalan que este tipo de manuales fueron una respuesta al contexto hostil hacia la práctica del ciclismo por parte de las mujeres quienes debieron desarrollar estrategias para utilizar la bicicleta bajo conductas consideradas apropiadas.

Algunas de las mujeres ciclistas de Buenos Aires asienten que el uso de los espacios públicos por parte de las mujeres continúa restringido, por lo mismo la utilización de la bicicleta es una situación donde se negocian sentidos y expectativas. Lo perciben en cada ocasión que alguien les sugiere que deberían circular en ciertos horarios, vestir de cierta forma, ir por ciertas calles, y, además en el actual contexto predominado por automóviles, también se experimentan situaciones de violencia vial. Aún habrá uno que otro manual para mujeres que quieren utilizar la bicicleta, pero, grupos como Mujeres sin Frenos se constituyen más como espacios sociales que se hacen en la práctica, desde los cuales reivindican la equidad del uso del espacio público a partir de ser mujeres y de la seguridad vial por ser ciclistas. Estos grupos de mujeres ciclistas buscan motivar que más mujeres utilicen las bicicletas, que ganen confianza al circular por las calles a partir de ir sumando experiencias individuales y colectivas, y es una manera de reivindicar el cuerpo femenino, de su presencia autónoma en las calles. 
Transitar por las ciudades es un desafío constante que se vive de distinta manera para cada persona. En este sentido, indico dos ejemplos dispares. Un ciclista, Ramiro, me comentó que reconoce que puede haber algunos sitios peligrosos de la ciudad, por lo que suele llevar sus herramientas en caso de que le suceda algo a su bicicleta y pueda repararla al instante para no estar mucho tiempo si es un sitio de riesgo, con esa actitud, él se mueve por toda la ciudad y a la hora que sea. Por otro lado, Laura, me dijo que ha aprendido a circular con precaución porque ha escuchado que han ocurrido agresiones hacia las mujeres que van más allá del simple acoso callejero, por eso prefiere las avenidas porque son más amplias e iluminadas. Lo que se puede notar en estos dos ejemplos, a riesgo de generalizar, es que si bien la ciudad puede ser peligrosa, es un desafío particular para las mujeres. No sólo las respuestas son distintas por la formación sesgada del género, sino que reflejan la carga de violencia hacia las mujeres. Este tipo de situaciones son las que agrupaciones como Mujeres sin Frenos intentan poner en evidencia entre mujeres para compartir otras maneras de enfrentar las situaciones cotidianas que viven al circular en bicicleta.

El uso de la bicicleta por parte de las mujeres que realizan paseos en grupo, además de herramienta emancipadora, retoma otros derechos desde la perspectiva de género. Es decir, aquellos derechos vinculados a las mujeres y también derechos como el de la movilidad, pero desde la visión de las mujeres. Estos paseos pueden relacionarse con un contexto más amplio de convergencia de ciudadanía de género que están impulsando las mujeres desde diferentes ámbitos, por ejemplo, la construcción de una planificación con perspectiva de género que retoma las formas en que las mujeres acceden a los entornos urbanos. Esta confluencia incluye críticas al sistema heteropatriarcal y al binomio sexual. En Argentina, igual que en otras ciudades latinoamericanas, hay presencia de otros grupos de mujeres ciclistas, o en Buenos Aires también hay grupos ciclistas de lesbianas, transexuales, queer, que reflejan la diversificación de la práctica del ciclismo y su flexibilidad para vincularse con otras reivindicaciones a la par de la movilidad.

\section{Concebir la ciudad desde paseos en bicicleta}

Dentro de los antecedentes inmediatos para el auge de la bicicleta en Buenos Aires habría dos acontecimientos, si bien son contrapuestos. El primero fue el paseo que realizaron un grupo de 22 ciclistas desde Plaza de Mayo en octubre del 2008, en aquella salida informaban que se trataba de una masa crítica, una idea trasladada de la Critical Mass de San Francisco (Carlsson, 2002), la cual fueron repitiendo los primeros domingos de cada mes realizándose sin interrupciones hasta la actualidad. El segundo acontecimiento proviene del gobierno de la ciudad del periodo 2007-2011 con el inicio de la construcción en el 2009 de la Red de Ciclovías Protegidas y el posterior seguimiento de la iniciativa con la implementación del sistema de transporte público en bicicleta Ecobici en el 2010 (Secretaría de Transporte, 2015). Hay otros antecedentes que indican algunas personas y organizaciones civiles, como la crisis económica del 2001 porque, dicen, propició que las personas utilizaran la bicicleta como medio de transporte.

El número de ciclistas en la ciudad ha aumentado, y la percepción hacia el ciclismo urbano también se ha modificado, la fuerza de la presencia de las bicicletas ha ido ganando terreno en las representaciones de esta práctica. En una cápsula informativa de televisión del 2011 las personas entrevistadas se mostraban renuentes a considerar la bicicleta como un medio de transporte eficaz, además de que la mayoría consideraba que les quitaba espacio para los automóviles, para los peatones, para los comercios. Apenas tres años después, en otra cápsula informativa del 2014, las personas indicaban que la bicicleta tenía beneficios en la salud y era considerada una buena opción para 
viajar en la ciudad. Por otro lado, como he indicado en la introducción, en el 2015 la ciudad de Buenos Aires fue enlistada dentro de las 20 ciudades mundiales más amigables para el ciclismo.

El auge de la práctica del ciclismo urbano pasa también por el surgimiento de diferentes colectivos y agrupaciones vinculadas al ciclismo, algunos están formalizados y otros simplemente son recreativos. Algunos de estos grupos organizan paseos ocasionales, abiertos a quienes quieran participar que muestran las distintas preferencias al practicar el ciclismo (en el apartado anterior he comentado características de un tipo de estos paseos, uno dedicado a mujeres). Los paseos colectivos en bicicleta son interacciones intencionadas de reunión y de transitar en bicicleta. En un trabajo exploratorio previo, a partir de participar en diferentes paseos que organizan los colectivos, se identificó que los paseos en bicicleta se pueden clasificar en: turísticos, oficialistas, de reconocimiento, y reivindicativos. Cada una de estas maneras de viajar por la ciudad implica diferentes relaciones sociales entre quienes participan y hacia los entornos urbanos.

Los paseos turísticos y oficialistas están organizados por el gobierno de la ciudad, los primeros por el ente de turismo que ha elaborado rutas "atractivas" que muestran algunos elementos de la ciudad a manera de identidad porteña. Los segundos se realizan aprovechando algunas fechas específicas que pueden capitalizarse con el uso de la bicicleta. En ambos casos, los paseos están restringidos a la infraestructura creada para promocionar el ciclismo urbano (ciclovías y bicisendas), están regulados por las disposiciones de tránsito (uso de casco, entre otras), y por otro lado, en los paseos oficialistas que ocupan las calzadas se genera una especie de cápsula alejada del flujo de los automóviles (se cierran calles), es decir, se aísla la práctica del ciclismo en relación a la interacción con los flujos urbanos o con los otros actores con quienes se comparten las calles.

El siguiente tipo de paseos son los de reconocimiento, aunque el objetivo de estos paseos está relacionado con realizar un trayecto y menos hacia referencias de los lugares por donde se transita, al realizar estos recorridos se abre la posibilidad de ampliar los mapas imaginarios de la ciudad o de observar los fragmentos de la ciudad por donde se pasa de una manera diferente (Van Duppen y Spierings, 2013). Estos paseos están organizados por agrupaciones que realizan recorridos en la ciudad, en el área metropolitana, o fuera de esta área. Estos grupos se dividen a su vez en los que realizan una planificación del recorrido e indican puntos de inicio, por donde pasarán y de finalización; y hay un colectivo específico que sólo tiene punto de inicio y fin sin predisponer el trayecto.

De aquellos que realizan una planificación del recorrido están Bicicleteadas Improvisadas y Recorridos Urbanos en Bicicleta, por indicar sólo un par, que gestionan paseos diurnos y nocturnos para disfrutar de la ciudad en grupo, una modalidad que ofrece seguridad a quienes lo integran, primero frente a los automóviles, en segundo lugar, por si sucede alguna avería con una bicicleta se ayudarían mutuamente, y en tercer lugar, en grupo pueden circular o recorrer lugares de la ciudad que de manera individual no se pasaría porque está fuera de las actividades cotidianas o porque se considere de riesgo.

El otro tipo de paseos que sólo tienen punto de inicio y fin es el realizado por el colectivo Masa Crítica Bs. As. Se trata de una manifestación ocasional que intenta celebrar la elección por el uso de la bicicleta como medio de transporte, su origen se sitúa en la ciudad de San Francisco en 1992 (Carlsson, 2002). En la ciudad de Buenos Aires se remonta a finales del 2007 cuando se realizó un primer intento de convocatoria, pero fue hasta octubre del 2008 que comenzaron a sumarse más personas. Desde entonces se realiza los primeros domingos de mes, y después, desde 2011 se ha agregado un paseo nocturno las noches de luna llena. Esta manifestación se realiza bajo pocas indicaciones, las principales son que no hay trayecto fijo ni una duración establecida, 
y durante el paseo mantener la densidad del grupo como un conjunto. Dependiendo de la asistencia de ciclistas (en 2012 se llegaron a los 3 mil asistentes) el cuerpo del colectivo es mayor o menor y provoca los respectivos cierres de calles sólo para un tipo de actores viales, porque, como lo indican durante la manifestación espontánea, "No bloqueamos el tráfico. Somos el tráfico". La página de Facebook del grupo es un foro y tablón de anuncios virtuales en el que se mantiene un sentido de comunidad, además de que en el espacio llamado Archivos están disponibles una variedad de documentos como leyes de tránsito, análisis sociológicos, hasta volantes de convocatorias de años anteriores. Destaca, por otro lado, el uso de una aplicación que utiliza GPS para telefonía móvil que sirve para indicar la ubicación propia y la de las demás participantes que también tengan activada la aplicación.

El último tipo de paseos son los reivindicativos, en los cuales se manifiesta la demanda del derecho a la movilidad y se comparte con otros propósitos derivados de las subjetividades y posicionamientos políticos. En este sentido, Masa Crítica Bs. As. es un ejemplo de traslape entre los tipos de paseos de reconocimiento y reivindicativos porque la única demanda que se realiza durante estos recorridos es el respeto hacia las y los ciclistas por parte de los demás actores viales para compartir las calles; por lo demás, se trata de un paseo de reconocimiento de la ciudad. Por otra parte, hay otros grupos que realizan otro conjunto de reivindicaciones, uno de estos lo he indicado en el apartado anterior, el de Mujeres sin Frenos, el cual ha venido conformando una posición política desde la perspectiva de género.

La particularidad de los paseos colectivos en general es que se realiza una interacción con los entornos urbanos distinta a los viajes que se realizan para las actividades cotidianas (Van Duppen y Spierings, 2013). Por ejemplo, en un paseo realizado en el barrio de Dorrego, uno de los asistentes, un hombre de 50 años, le comentaba con sorpresa a su pareja que había escuchado del edificio que estábamos viendo, el Tanque distribuidor Devoto, pero que no lo había visto ni sabía con exactitud en dónde estaba. En este sentido, la ciudad se va construyendo a partir del reconocimiento que se realiza en cada viaje, y en este caso, con los paseos porque amplían o confirman las imágenes que se tienen de la ciudad y, además, del intercambio de las experiencias entre las personas participantes. En varias ocasiones estuve en medio de personas que comentaban algunas rutas que toman desde un punto $\mathrm{A}$ hacia uno $\mathrm{B}$, de las condiciones de las calzadas, de horarios más convenientes para circular, o de nuevos lugares que visitar; todos esos intercambios evidenciaban un uso constante de la bicicleta para moverse a través de la ciudad (Švarc, 2017).

Durante los paseos sentía una sensación de circular por donde realmente quería ir, similar a las líneas de deseo (originalmente "desire paths" en Bachelard, 1958). Es decir, podía transitar por la ciudad junto con las demás personas ocupando un carril de la calzada sin preocuparme de los otros actores viales, porque conformábamos un cuerpo que les hacía esperar o moverse por los costados de nosotros. Esto no implicaba que uno se descuidara del flujo vial, o de los reglamentos de tránsito, sino que había una sensación de seguridad distinta a cuando viajaba sólo en bicicleta. Sumado a ello, el ambiente que se genera entre las personas participantes es ameno, cordial, no hay prisa por llegar a un sitio si bien se mantiene una velocidad. También iba identificando la manera como las interacciones con las demás personas propician, en algunas, relaciones a largo plazo, lo veía en las personas asistentes al saludarse, preguntarse la ruta a seguir después del paseo, de cuidarse entre el grupo. La constante asistencia a estos paseos termina por estrechar algunas relaciones.

Hay otro tipo de paseos para conocer y reconocer la ciudad que pueden realizarse con los grupos, pero sin asistir a los recorridos, se trata de los registros audiovisuales que asistentes realizan durante los paseos y que posteriormente comparten en las redes 
sociales virtuales. Un ejemplo es la página de Facebook de Ciclismo Urbano que tiene una gran cantidad de álbumes donde muestran sus perspectivas de los entornos urbanos que han recorrido, algunas imágenes son de puntos poco observados mientras que otros son fácilmente reconocibles. Otro ejemplo son los centenares de fotografías y videos que los asistentes a la Masa Crítica comparten en cada paseo, así como los trayectos que han realizado. En este sentido, los comentarios de los usuarios de redes sociales también son parte de esos intercambios que brindan información de los lugares, los recorridos y de los barrios.

En los paseos colectivos puede observarse una mayor participación de mujeres, que contrasta con la menor presencia de mujeres ciclistas en el resto de la ciudad, es decir, para las actividades cotidianas. Como indican Aldred, Woodcock y Goodman (2016), el incremento del ciclismo no implica necesariamente que exista una distribución de la población en el uso de la bicicleta, por ejemplo, en ciudades que comienzan a presentar mayor uso de la bicicleta hay predominio de hombres, y por generación suelen ser jóvenes y adultos (Aldred, Woodcock y Goodman, 2016:32-33). Pero, lo que yo fui observando es que había una variación entre las personas que utilizan la bicicleta como medio de transporte para las actividades cotidianas, donde había menos mujeres, mientras que en los paseos observaba una mayor diversidad, quizá porque era una situación que reunía o convocaba, precisamente, a esa diversidad.

Un aspecto que ha permitido a la bicicleta ser el nuevo objeto (Vivanco, 2013) es precisamente la flexibilidad de la práctica y de la bicicleta misma para trasladarnos hacia cualquier parte y transportarla si es necesario. Pero, quizá la confluencia de reivindicaciones hacia el uso del espacio público, hacia la equidad vial, o la pacificación vial, sean los elementos que estén haciendo de la bicicleta el objeto ideal para posicionarnos políticamente ante los cambios que están sucediendo en las ciudades. Precisamente, una entrada del blog de Ciclofamilia dice "la gente siente que conquista un espacio de la ciudad" (Espíndola, 2016), en relación a la sensación de las personas en un paseo dirigido a generar confianza, desarrollar destrezas para andar en bicicleta y puedan incorporarla en sus opciones de transporte. Ante los contextos urbanos donde predomina el diseño y planificación pensada desde el automóvil (Edensor, 2004; Hall, 2003), cada persona que comienza a utilizar la bicicleta participa en un movimiento que amplía la práctica social del ciclismo urbano (Larsen, 2016) y que se dirige a la equidad del uso del espacio público.

\section{Transitando hacia las políticas públicas}

Durante el mes de junio del 2017 una organización civil lanzó una convocatoria para atender un asunto emergente. En la legislatura porteña se debatiría sobre la prohibición del tránsito de las bicicletas en las avenidas, se trataba de una iniciativa lanzada por un diputado local. Así, un sábado en la noche nos reunimos las personas que nos sentimos interpeladas para conocer detalles de la propuesta y de posibles acciones a realizar. El siguiente lunes acudimos a la sesión y algunas personas vinculadas al ciclismo urbano se pronunciaron ante dicha propuesta de prohibición. No habían acudido muchas personas, pero las que asistieron dieron argumentos sólidos relacionados a la movilidad como un derecho y a la práctica del ciclismo como una necesidad para la ciudad. Esta situación de emergencia se repitió una segunda ocasión en el mismo mes porque se debatiría, en esa oportunidad, una propuesta para restringir un límite de velocidad para los ciclistas.

Aquellas ocasiones que la legislatura porteña intentó restringir la práctica del ciclismo urbano, pusieron en evidencia que con todo y políticas públicas que intentan incentivar 
el uso de la bicicleta, el derecho a la movilidad continúa siendo una exigencia para quienes hemos decidido utilizar la bicicleta como medio de transporte. La manera como los centros, colectivos y agrupaciones vinculadas al ciclismo han encontrado para generar apoyos en torno a la bicicleta como una herramienta para democratizar el uso del espacio público, es combinar otras demandas ciudadanas o formas de hacer ciudad, comunidad, colectividad, por ejemplo, los talleres populares de ciclomecánica que tienen larga tradición en la ciudad.

En este sentido, el ciclismo urbano ha generado un ambiente propicio para el tejido social, muestra de ello son las diferentes organizaciones civiles como Ciclofamilia o la Asociación de Ciclistas Urbanos. A la vez, un ejemplo de una acción grupal es la solicitud realizada a principios del 2017 por parte de 33 organizaciones civiles de todo el país, el colectivo Argentina en Bici (ABi), hacia todos los niveles de gobierno del país para que comenzaran a implementar una serie de premisas de corto plazo para aminorar los siniestros viales: Bajar la velocidad en entornos urbanos; alcohol cero al volante o manubrio; celular cero al volante o manubrio; respeto al ciclista en adelantamientos (mantener 1.5mts de distancia); optimización de estadística; y bicicletas blancas.

Una acción simbólica que sobresale desde las demandas ciudadanas es la colocación de memoriales para los fallecimientos por causas viales, para peatones y para ciclistas: estrellas amarillas y bicicletas blancas. Por parte de los colectivos ciclistas se organizan para realizar un acto en el cual se dirigen al lugar donde ocurrió el accidente, llevan una bicicleta pintada de blanco y la colocan en la vía pública. La intención es generar un recuerdo material y permanente, un testimonio de un accidente vial donde estuvo involucrado un ciclista. Sin embargo, la violencia vial es constante.

En noviembre del 2016, un día cualquiera de la semana, en la equina de Av. Entre Ríos y la calle México, al esperar el cambio de semáforo observé un choque entre un automovilista y un motociclista, el primero iba en exceso de velocidad e intentó cruzar en amarrillo mientras que el segundo se adelantó unos segundos al cambio de luces. Unos minutos después, pero en Av. Callao y la calle Bartolomé Mitre, ocurrió otro choque, en ese caso entre dos automovilistas que habían repetido el mismo patrón del anterior, uno quería llegar al cambio de luces y el otro se había adelantado unos segundos. Aquella tarde fue, primero, impactante por haber presenciado a unos metros ambos accidentes en el transcurso de sólo unos minutos, y después, reveladora sobre el nivel de estrés y violencia vial que existe entre quienes conducen vehículos motorizados.

Otra de las propuestas que se realizan desde la sociedad, algunas veces desde los foros virtuales de las redes sociales y otras veces desde las voces de las organizaciones civiles, es que las personas involucradas en la gestión y reformas de políticas públicas, del diseño y planificación urbana relacionada al ciclismo, deberían realizar recorridos en bicicleta por la ciudad. Esto les permitiría observar desde sus vivencias las situaciones cotidianas que se tienen que sobrellevar día a día. Por ejemplo, en una plática con Silvia, una ciclista urbana, me decía que justo entre las calles Chiclana y Deán Funes hay unos puntos ciegos que ella tiene que sortear y, según su observación, podrían resolverse de manera simple con un trazado distinto de la ciclovía. Pero, este no fue el único caso, había muchos más, entre el más comentado está el de la calle Perón.

Finalmente, una de las exigencias recurrentes por parte de integrantes de los diferentes colectivos y agrupaciones vinculadas al ciclismo es la de reconocer que en los momentos actuales existe inequidad en la distribución y el uso del espacio urbano. Reconocerlo ayudaría a orientar las políticas públicas vinculadas al tránsito y los transportes sustentables. En la siguiente figura se puede observar de manera más clara la distribución actual del uso del espacio urbano en relación al transporte y la velocidad. 


\begin{tabular}{llll} 
& & \\
\hline
\end{tabular}

Figura 1. Espacio urbano utilizado por las personas de acuerdo al medio de transporte y velocidad. Fuente: Urban Mobility Plan Vienna. Together on the Move. Step 2025, 2014:48.

\section{Conclusiones}

Podemos identificar que desde la invención de la bicicleta y su expansión el ciclismo ha estado presente en las calles, así lo pone en evidencia algún manual del siglo XIX (Erskine, 1897; Ward, 1896; Willard, 1895), alguna fotografía antigua, algún relato de personas mayores, incluso nuestros referentes inmediatos en torno a este objeto. Sin embargo, a pesar de que estamos rodeados de bicicletas (Horton, Cox y Rosen, 2007) o que inevitablemente tengamos alguna historia o anécdota propia (Augé, 2009), la bicicleta y el ciclismo urbano aún son considerados temas nuevos para las dinámicas de las ciudades, aún más, las y los ciclistas urbanos aún debemos hacer valer el derecho a la movilidad (Golub et al., 2016). Por otro lado, las relaciones y los procesos sociales que se dan en los momentos actuales en torno a la bicicleta tienen algo de novedoso (Vivanco, 2013).

En este texto se puede apreciar que el ciclismo urbano es una práctica específica de movilidad por la ciudad y, principalmente, que es heterogénea. Así, un aspecto a destacar es el surgimiento de espacios de socialización en torno a los paseos colectivos en bicicleta. Estos espacios tienen la particularidad de que se conforman en el momento que se va transitando por la ciudad. Además, al realizarse en una situación de interacción específica abren la posibilidad de ampliar los conocimientos que se tienen de la ciudad, al mismo tiempo que se retroalimentan las experiencias individuales y colectivas. También generan impactos en el uso del espacio y de lo público los grupos de ciclistas, no sólo por su tránsito sino por las demandas que construyen.

Tal como se reconoció en varias pláticas y desde la experiencia propia, es distinto saber andar en bicicleta a practicar el ciclismo urbano. Para participar en los paseos colectivos y para transitar por la ciudad en bicicleta, es necesario un proceso de adaptación en el cual se van cambiando las técnicas corporales y se ajustan los componentes de la bicicleta para hacer un acoplamiento que nos resulte cómodo para mantener el equilibrio y el pedaleo. Además, acumular una serie de conocimientos relacionados a los reglamentos de tránsito, tener el equipamiento básico de mantenimiento y de seguridad, e intercambiar experiencias con otras y otros ciclistas.

Por otro lado, se debe hacer patente que la bicicleta, por más versátil que sea, no puede resolver los problemas relacionados al tránsito en las ciudades, ni los de contaminación. 
Pero, sí ofrece posibilidades muy variadas para complementar políticas de sustentabilidad que pueden adaptarse a las características de cada ciudad. Así, la lucha por la equidad en el espacio público no es por eliminar actores viales (automóviles) sino de maneras de compartir el espacio y los flujos viales.

Para terminar este artículo, retomo la provocación que hacen las integrantes del Col-lectiu Punt 6 de Barcelona para repensar los accesos y usos de los entornos urbanos: pensar en las personas que no se ven en la ciudad. Para el caso del ciclismo valdría preguntarnos quiénes son esas personas que no utilizan la bicicleta como medio de transporte, cuáles serán sus razones y percepciones por las cuales aún no pedalean una bici.

\section{Agradecimientos}

La realización de esta investigación fue posible a la beca de estancias posdoctorales al extranjero del Consejo Nacional de Ciencia y Tecnología (Conacyt) de México, la cual fue realizada en el Instituto de Investigaciones Gino Germani de la UBA durante el periodo 2016-2017. El agradecimiento es para lxs integrantes de la Asociación de Ciclistas Urbanos (ACU) de Buenos Aires, quienes mantuvieron abiertos los espacios para el intercambio, también a la agrupación Ciclofamilia por la generosidad en los aprendizajes (siempre necesarios) para rodar la ciudad. Un agradecimiento especial a todas las personas ciclistas de Buenos Aires por compartir sus experiencias. 


\section{Q Bibliografía}

" ACU y EN BICl SEGURO, Asociación de Ciclistas Urbanos y En Bici Seguro (2013). Guía del ciclista urbano. Buenos Aires: ACU y En Bici Seguro.

» Aldred, R. (2013). Incompetent or Too Competent? Negotiating Everyday Cycling Identities in a Motor Dominated Society. Mobilities, vol. 8 n 2, pp. 252-271.

"Aldred, R. (2015). A Matter of Utility? Rationalising Cycling, Cycling Rationalities. Mobilities, vol. 10 ํㅜ 5, pp. 686-705.

" Aldred, R., Woodcock, J., Goodman, A. (2016). Does More Cycling Mean More Diversity in Cycling? Transport Reviews, vol 36 n 1, pp. 28-44.

"Augé, M. (2009). Elogio de la bicicleta. Barcelona: Gedisa.

» Bachelard, G. (1958). The Poetics of Space. Boston: Beacon Press.

» Banco Interamericano de Desarrollo (2015). Ciclo-Inclusión en América Latina y el Caribe. Guía para impulsar el uso de la bicicleta. BID.

»Caballero, R., Franco, P., Mustaca, A., Jakovcevic, A. (2014). Uso de la bicicleta como medio de transporte: influencia de los aspectos psicológicos. Una revisión de la literatura. Psico, vol. 45 n³, pp. 316-324.

»Carlsson, C. (Ed.) (2002). Critical Mass: Bycicling's Defiant Celebration. Oakland: AK Press.

»Coghlan, A. (2012). An autoethnographic account of a cycling charity challenge event: Exploring manifest and latent aspects of the experience. Journal of Sport E Tourism, vol. 17 no 2, pp. 105-124.

»Copenhagenize Design (2013). The Bicycle Choreography of an Urban Intersection. Frederiksberg: Copenhagenize Design Co.

"Csordas, T. (1994). Introduction: the body as representation and being-in-theworld. En: Embodiment and Experience. The existential ground of culture and self. Csordas, T. (ed.) Cambridge: Cambridge University Press, pp. 1-24.

»DPCABA, Defensoría del Pueblo Ciudad Autónoma de Buenos Aires (2017). Datos sobre siniestros viales año 2016. Informe estadístico. Buenos Aires: DPCABA.

»Edensor, T. (2004). Automobility and National Identity. Representation, Geography and Driving Practices. Theory, Culture \& Society, vol. 21 no 4/5, pp. 101-120.

»Ellis, C., Bochner, A. P. (2000). Autoethnography, Personal Narrative, Reflexivity: Researcher as Subject. En: Handbook of Qualitative Research, Denzin, N., Lincoln, Y. (eds.) Thousand, Oaks: Sage, pp. 733-768.

"Erskine, F. J. (1897). Lady Cycling - What to Wear and How to Ride. Londres: Walter Scott Ltd.

»Espíndola, M. (2016). La gente siente que conquista un espacio de la ciudad. Ciclofamilia, 23 de noviembre, <http://ciclofamilia.com.ar/la-gente-siente-queconquista-un-nuevo-espacio-en-la-ciudad/>

» Golub, A., Hoffmann, M., Lugo, A., Sandoval, G. (eds.) (2016). Bicycle Justice and Urban Transformation. Biking for all? Londres y Nueva York: Routledge.

» Hall, T. (2003). Car-ceral Cities: Social Geographies of Everyday Urban Mobility. 
En: Urban Futures: Critical Commentaries on Shaping the City, Miles, M. y Hall, T. (eds.). Londres: Routledge, pp. 92-105.

" Herlihy, D. (2004). Bicycle: The History. New Haven: Yale University Press.

» Horton, D., Cox, P., Rosen, P. (2007). Introduction: Cycling and Society. En: Cycling and Society, Horton, D., Rosen, P. y Cox, P. (eds.), Burlington: Ashgate Publishing, pp. 1-23.

» Huerta, E., Gálvez, C. (2016). Mujeres en bicicleta. Imaginarios, prácticas y construcción social del entorno en la ciudad de Sevilla. Revista de Antropología Experimental, no 16, pp. 111-128. http://revistaselectronicas.ujaen.es/index.php/ rae/article/view/3020

» Jones, P. (2005). Performing the city: a body and a bicycle take on Birmingham, UK. Social \& Cultural Geography, vol.6 no 6, pp. 813-830.

" Jordi Sánchez, M. (2016). Bicicleta y corporalidades. Salud, imagen y sensaciones tecnocorporales en la bicicleta. En VV. AA. Bicitopías. Hacia una antropología de la movilidad urbana. Sevilla: Universidad Pablo Olavide e Icaria editorial, pp. 209-239.

"Larsen, J. (2016). The making of a pro-cycling city: Social practices and bicycle mobilities. Environment and Planning A, vol. o, pp. 1-17.

» Larsen, J. (2014). (Auto)Ethnography and cycling. International Journal of Social Research Methodology, 17 (1), 59-71.

»Latham, A., Wood,P. (2015). Inhabiting infrastructure: exploring the interactional spaces of urban cycling. Environment and Planning A, 47, 300-319.

»Lorenc, T., Brunton, G., Oliver, S., Oliver, K., Oakley, A. (2008). Attitudes to walking and cycling among children, young people and parents: a systematic review. Journal of Epidemiology \& Community Health, 62 (10), 852-857.

»Lugo, A. (2012). Planning for Diverse Use/rs: Ethnographic Research on Bikes, Bodies, and Public Space in Los Angeles. Kroeber Anthropological Society Papers 101, 49-65.

» Lugo, A. (2013). CicLAvia and human infrastructure in Los Angeles: ethnographic experiments in equitable bike planning. Journal of Transport Geography, 30, 202-207.

» Lugo, A., Mattheis, A., Aguirre, M. (2017). Bicycle Anthropology of Los Angeles. En: Anthropology of Los Angeles: Place and Agency in an Urban Setting, Banh, J. y King, M. (eds.), Lanham: Lexintong Books, pp. 21-43.

" Mackintosh, P. y Norcliffe, G. (2007). Men, Women, and the Bicycle: Gender and Social Geography of Cycling in the Late Nineteenth Century. En: Cycling and Society. Horton, D., Rosen, P. y Cox, P. (eds.). Burlington: Ashgate Publishing, pp. 153-178.

» Mauss, M. (1934). Les techniques du corps. Sociologie et Anthropologie. París: Presses Universitaires de France, 1950.

»Parkin, J., Ryley, T., Jones, T. (2007). Barriers to Cycling: An Exploration of Quantitative Analyses. En: Cycling and Society. Horton, D., Rosen, P. y Cox, P. (eds.). Burlington: Ashgate Publishing, pp. 67-82.

"Penn, R. (2011). It's All About the Bike: The Pursuit of Happiness on Two Wheels. New York: Bloomsbury USA.

"Secretaría de Transporte (2017). Manual del ciclista. Buenos Aires: Gobierno de la Ciudad de Buenos Aires.

»Secretaría de Transporte (2015). Ecobici. La historia de la bici en Buenos Aires. Buenos Aires: Gobierno de la Ciudad de Buenos Aires. 
»Sheller, M., Urry, J. (2006) The new mobilities paradigm. Environment and Planning A, 38, 207-226.

" Simpson, P. (2016). A sense of the cycling environment: Felt experiences of infrastructure and atmospheres. Environment and Planning A, vol. o, pp. 1-22.

"Spinney, J. (2009). Cycling the city: movement, meaning and method. Geography Compass, 3 (2), 817-835.

» Švarc, M. (2017). Movement, body and practice: Understanding everyday practices of bicycling. Tesis de master en Sociología, Universidad Masaryk.

» Turner, V., Bruner, E. (eds.) (1986) The Anthropology of Experience. Chicago: University of Illinois Press.

"Van Duppen, J., Spierings, B. (2013). Retracing trajectories: the embodied experience of cycling, urban sensescapes and the commute between 'neighbourhood' and 'city' in Utrecht, NL. Journal of Transport Geography 30, 234-243.

»Vivanco, L. (2013). Reconsidering the Bicycle. An Anthropological Perspective on a New (Old) Thing. Nueva York y Oxon: Routledge.

"Ward, M. (1896). Bicycling for ladies. With hints as to the art of wheeling-Advice to beginners-Dress-Care of the bicycle-mechanics-Training-Excercise, etc.,etc. Nueva York: Bretano's.

"Whitt, F., Wilson, D. (1982) Bicycling Science. Cambridge, MA.: MIT Press, 1995.

"Willard, F. (1895). A Wheel Within a Wheel - How I Learned to Ride the Bicycle with Some Reflections by the Way. Toronto: Fleming H. Revell Co.

"Wright, P. (2013). Imaginarios, símbolos y coreografías viales: una perspectiva antropológica. Revista Seguridad Vial, 121, 1-6.

» Zunino Singh, D. (2014). Meaningful Mobilities: The Experience of Underground Travel in the Buenos Aires Subte (1913-1944). Journal of Transport History, 35 (1), 97-113.

\section{Oscar Ramos Mancilla / oscar.orm@gmail.com}

Doctor en Antropología social (Universitat de Barcelona). Investigador posdoctoral (IIGG-UBA). Se interesa en la cultura digital, las metodologías y etnografías experimentales, las movilidades, y los procesos socio-históricos. Por sus investigaciones ha obtenido: el premio Sor Juana Inés de la Cruz del Inmujeres (2008), el premio Noemí Quezada del Coloquio Internacional sobre Otopames (2011), una mención honorífica en el premio Fray Bernardino de Sahagún del INAH (2012), y el premio de la Cátedra Bi-Institucional Gonzalo Aguirre Beltrán del CIESAS y la UV (2017). 